\title{
Early neurodevelopmental outcome in preterm posthemorrhagic ventricular dilatation and hydrocephalus: Neonatal ICU Network Neurobehavioral Scale and imaging predict 3-6-month motor quotients and Capute Scales
}

\author{
Rebecca A. Dorner, MD, MHS, ,1,2 Marilee C. Allen, MD, ,,2 Shenandoah Robinson, MD, ,2,3 \\ Bruno P. Soares, MD, ${ }^{4}$ Jamie Perin, PhD, ${ }^{5}$ Ezequiel Ramos, ${ }^{6}$ Gwendolyn Gerner, PsyD, ${ }^{8}$ and \\ Vera Joanna Burton, MD, $\mathrm{PhD}^{7,9}$ \\ ${ }^{1}$ Division of Perinatal-Neonatal Medicine, Department of Pediatrics, ${ }^{3}$ Pediatric Neurosurgery, and ${ }^{9}$ Department of Neurology, \\ Johns Hopkins University School of Medicine; ${ }^{2}$ Neurosciences Intensive Care Nursery, Johns Hopkins Hospital, Baltimore, \\ Maryland; "Radiology, University of Vermont Medical Center, Burlington, Vermont; ${ }^{5}$ Bloomberg School of Public Health, \\ Biostatistics, Epidemiology, and Data Management Core, Johns Hopkins University, Baltimore, Maryland; ${ }^{6}$ Medical Doctorate \\ Program, Icahn School of Medicine at Mount Sinai, New York, New York; and ${ }^{7}$ Neurology and Developmental Medicine and \\ ${ }^{8}$ Neuropsychology, Kennedy Krieger Institute, Baltimore, Maryland
}

OBJECTIVE Brain injury remains a serious complication of prematurity. Almost half of infants with severe intraventricular hemorrhage (IVH) develop posthemorrhagic ventricular dilatation (PHVD) and 20\% need surgery for posthemorrhagic hydrocephalus $(\mathrm{PHH})$. This population is associated with an increased risk of later neurodevelopmental disability, but there is uncertainty about which radiological and examination features predict later disability. In this study the authors sought to devise and describe a novel combination of neurobehavioral examination and imaging for prediction of neurodevelopmental disability among preterm infants with PHVD and $\mathrm{PHH}$.

METHODS The study patients were preterm infants ( $<36$ weeks gestation) with IVH and PHVD, with or without $\mathrm{PHH}$. Ventricular index (VI), anterior horn width (AHW), thalamooccipital distance (TOD), ventricle/brain (V/B) ratio, and resistive indices (RIs) were recorded on the head ultrasound (HUS) just prior to surgery, or the HUS capturing the worst PHVD when surgery was not indicated. The posterior fossa was assessed with MRI. Neonatal ICU Network Neurobehavioral Scale (NNNS) examinations were performed at term age equivalent for each infant. A neurodevelopmental assessment using the Capute Scales (Capute Cognitive Adaptive Test [CAT] scores and Capute Clinical Linguistic Auditory Milestone Scale [CLAMS] scores) and a motor quotient (MQ) assessment were performed between 3 and 6 months of age corrected for degree of prematurity (corrected age). MQs $<50$ reflect moderate to severe delays in early motor milestone attainment, CAT scores $<85$ reflect delays in early visual and problem-solving abilities, and CLAMS scores $<85$ reflect delays in early language.

RESULTS Twenty-one infants underwent assessments that included imaging and NNNS examinations, Capute Scales assessments, and MQs. NNNS nonoptimal reflexes (NOR) and hypertonicity subscores and AHW were associated with $\mathrm{MQs}<50$ : NOR subscore OR $2.46(95 \% \mathrm{Cl} 1.15-37.6, \mathrm{p}=0.034)$, hypertonicity subscore OR $1.68(95 \% \mathrm{Cl} 1.04-3.78$, $p=0.037)$, and AHW OR $1.13(95 \% \mathrm{Cl} 1.01-1.39, p=0.041)$. PVHI, cystic changes, and neurosurgical intervention were associated with CAT scores < 85: PVHI OR $9.2(95 \% \mathrm{Cl} 1.2-73.2, \mathrm{p}=0.037)$; cystic changes OR $12.0(95 \% \mathrm{Cl}$ $1.0-141.3, p=0.048)$, and neurosurgical intervention OR $11.2(95 \% \mathrm{Cl} 1.0-120.4, p=0.046)$. Every $1-S D$ increase in

ABBREVIATIONS AHW = anterior horn width; AP = anteroposterior; BPD = bronchopulmonary dysplasia; CAT = Capute Cognitive Adaptive Test; CLAMS = Capute Clinical Linguistic Auditory Milestone Scale; corrected age = age corrected for degree of prematurity; $\mathrm{CP}=$ cerebral palsy; $\mathrm{DQ}=$ developmental quotient; $\mathrm{HUS}=$ head ultrasound; $\mathrm{IVH}=$ intraventricular hemorrhage; $\mathrm{MQ}=$ motor quotient; NEC = necrotizing enterocolitis; NICU = neonatal ICU; NNNS = NICU Network Neurobehavioral Scale; NOR = nonoptimal reflexes subscore; $\mathrm{PHH}=$ posthemorrhagic hydrocephalus; $\mathrm{PHVD}=$ posthemorrhagic ventricular dilatation; $\mathrm{PMA}=$ postmenstrual age; $\mathrm{PVHI}=$ periventricular hemorrhagic infarction; $P V L=$ periventricular leukomalacia; $\mathrm{RI}=$ resistive index; $\mathrm{ROP}=$ retinopathy of prematurity; $\mathrm{TOD}=$ thalamooccipital distance; $\mathrm{V} / \mathrm{B}=\mathrm{ventricle} / \mathrm{brain}$; $\mathrm{VI}=$ ventricular index

SUBMITTED July 26, 2019. ACCEPTED September 16, 2019

INCLUDE WHEN CITING Published online December 20, 2019; DOI: 10.3171/2019.9.PEDS19438. 
the NOR subscore was associated with an increase in odds of a CAT score $<85$, OR $4.0(95 \% \mathrm{Cl} 1.0-15.0, p=0.044)$. Worse NNNS NOR subscores were associated with early language delay: for a 1-SD increase in NOR subscore, there was an increase in the odds of a CLAMS score < 85, OR $19.5(95 \% \mathrm{Cl} 1.3-303, \mathrm{p}=0.034)$.

CONCLUSIONS In former preterm children with severe IVH and PHVD, neonatal neurological examination findings and imaging features are associated with delays at 3-6 months in motor milestones, visual and problem-solving abilities, and language.

https://thejns.org/doi/abs/10.3171/2019.9.PEDS19438

KEYWORDS outcome; neurodevelopment; posthemorrhagic ventricular dilatation; posthemorrhagic hydrocephalus; assessment

$\mathrm{D}$ ESPITE advances in neonatal care, brain injury remains a serious complication of prematurity. Intraventricular hemorrhage (IVH) occurs in up to $30 \%$ of premature infants. Among infants with severe IVH, one-third to one-half develop posthemorrhagic ventricular dilatation (PHVD), and 20\% require surgical intervention due to posthemorrhagic hydrocephalus (PHH). ${ }^{3,41}$ Preterm infants with PHVD, particularly those requiring intervention for hydrocephalus, are at high risk for future neurodevelopmental disability, given comorbid and cumulative risks from IVH, ventriculomegaly, and white matter injury.

The spectrum of motor and cognitive sequelae includes cerebral palsy (CP), developmental coordination disorder, intellectual disability, memory and executive function deficits, attention deficit/hyperactivity disorder, cortical visual impairment, epilepsy, chronic pain, and behavior problems. ${ }^{9,21,23,25,35,42,43,45}$

Despite the magnitude and heterogeneity of these neurodevelopmental complications, there are important limitations in the literature regarding the measurement of motor and cognitive outcomes in patients with PHVD and PHH. These include inadequate delineation of PHVD from PHH and differing timings and types of neurodevelopmental assessments.

Larger lateral ventricles and more severe or higher grades of IVH in preterm infants have been associated with worse motor and cognitive scores. ${ }^{9,18,20,32,38}$ However, the current practice paradigm cannot delineate PHVD from $\mathrm{PHH}$ because it excludes precise measurements of both ventricular size and individual clinical information. This lack of delineation is important because preterm infants with $\mathrm{PHH}$ are at higher risk for gross motor abnormalities such as $\mathrm{CP}$ than preterm infants with IVH alone or PHVD without PHH., ${ }^{2,16,25}$

In addition, developmental assessments in this cohort are typically conducted after discharge from the neonatal ICU (NICU) and may consist only of parent reports of developmental milestone acquisition. ${ }^{50}$ This contributes to the varying reported types and frequencies of neurodevelopmental impairment in pediatric patients with $\mathrm{PHH} .{ }^{9,21,25}$

Although the need for interventions for brain injury and coexisting medical morbidities are closely tied to outcomes in preterm infants with $\mathrm{PHH}^{5,34}$ there are currently no specific imaging parameters that can be used to consistently predict outcomes in these patients. The use of evidenced-based neonatal assessment to quantify deficits in the neonatal period is needed both to augment existing clinical imaging practices and to develop and test targeted early interventions for infants with PHVD and $\mathrm{PHH}$ in clinical trials.

In the first study with this prospective cohort, motor and cognitive abnormalities identified on neonatal neurobehavioral examinations (NICU Network Neurobehavioral Scale [NNNS]) in infants with PHVD correlated with head ultrasound (HUS) and MRI findings. ${ }^{17}$ Specifically, higher NNNS nonoptimal reflexes (NOR) and hypotonicity subscores and lower attention subscores were associated with larger ventricles. Decreased habituation, or a limited ability to ignore repetitive stimuli, was associated with periventricular hemorrhagic infarction (PVHI).

The aim of our present study was to devise and describe a novel combination of developmental assessment tools and imaging modalities to improve prediction of early developmental deficits in infants with PHVD and PHH. In addition to the neonatal NNNS and brain imaging studies, we also performed comprehensive 3-6-month follow-up assessments for these same infants using motor quotients (MQs) and the Capute Scales. MQs quantify early gross motor delay by comparison of an infant's "motor age," or the age equivalent of their best motor performance on a series of typical motor milestones, to their "actual age" (in this case we used age corrected for degree of prematurity [corrected age]). ${ }^{12}$ The Capute Scales, a valid, reliable, and efficient 100-item developmental assessment tool, includes 2 subscales that measure visual and nonverbal problem-solving skills (Capute Adaptive Test [CAT]) and expressive and receptive language (Capute Linguistic and Auditory Milestone Scale [CLAMS]).10,33,46

\section{Methods \\ Study Design and Subjects}

Recruitment for this prospective cohort study was approved by the internal review board at Johns Hopkins Hospital and the Kennedy Krieger Institute and was conducted from July 1, 2016, to July 31, 2018. Eligible patients were preterm infants who were born during this time frame with IVH and ventricular dilatation, with or without $\mathrm{PHH}$, and were born in or referred to our level IIIB or IIIC NICUs. Infants with IVH and ventricular dilatation were classified as having PHVD. The subset of children within this group of PHVD patients who required neurosurgical intervention for hydrocephalus were classified as PHH. After the initial classification, no cases of PHVD were later classified as $\mathrm{PHH}$. The pediatric neurosurgeons at 
Johns Hopkins Hospital used the Hydrocephalus Clinical Research Network consensus guidelines regarding timing for surgical treatment. ${ }^{49}$ Consensus was reached according to these guidelines among the neurosurgery team for each individual infant. Infants were excluded if they had suspected or confirmed genetic anomalies. Eligible infants were identified via neurodevelopmental consultation with the Neurosciences Intensive Care Nursery or by request of the clinical team as part of routine NICU clinical practice for infants with IVH and PHVD. After NNNS examination was performed for clinical purposes, consent was obtained from parents or legal guardians to enroll the infant as a participant in this prospective clinical research cohort, for which participation included review of clinically obtained standard-of-care neuroimaging and neurodevelopmental follow-up examinations.

A total of 28 eligible infants were initially recruited, and for 21 of them data from complete neurodevelopmental evaluation at 3-6 months corrected age was available at the time of this report. The remaining 7 infants were lost to follow-up. For each eligible infant we recorded baseline demographic and comorbidity data, including IVH grade by the Papile scoring system, ${ }^{39}$ sepsis, necrotizing enterocolitis (NEC), bronchopulmonary dysplasia (BPD) severity, ${ }^{27}$ and retinopathy of prematurity (ROP).

\section{Neonatal Neurobehavioral Measures}

The NNNS examination is a standardized assessment tool that measures both neurological integrity and behavioral function and also includes a separate stress/abstinence scale to document stress behavior and state regulation through withdrawal. ${ }^{31}$ This examination has strong psychometric qualities and validity in the assessment of motor and cognitive deficits in the neonatal period..$^{37}$ Examiner certification requires formal instruction and reliability testing. The 2 examiners in this study (R.A.D. and V.J.B.) were independently certified and passed reliability testing with each other. The NNNS examinations were performed at the closest time point to term-age equivalent, ideally no sooner than 34 weeks postmenstrual age (PMA) and at least 10 days from the neurosurgical procedure, as previously described. ${ }^{17}$ These examinations were only performed in medically stable infants with the agreement of the treating medical team. The NNNS summary scores were grouped into the following 2 categories: motor (with subscores for hypertonicity, hypotonicity, excitability, lethargy, NOR, asymmetrical reflexes, and quality of movement) and cognitive (with subscores for habituation, attention, handling, regulation, stress/abstinence, and arousal).

The NNNS NOR subscore was used to assess the presence of newborn reflexes and strength of each response. The included reflexes are lower-extremity reflexes (plantar grasp, Babinski, elicited ankle clonus), upper-extremity grasp, oromotor reflexes (rooting, sucking), reflexes elicited with infant in prone position (crawling reflex and head raise), reflexes elicited with the infant in supine position (asymmetrical tonic neck reflex), reflexes elicited with the infant held in ventral suspension (incurvation), reflexes elicited with the infant held upright (placing, stepping), and reflexes elicited when spinning the infant (tonic devia- tion of the head and eyes and nystagmus). Results were recorded and analyzed using R: A Language and Environment for Statistical Computing (R Foundation for Statistical Computing). These reflexes differ with regard to the criteria for optimal responses; a brisk response is desirable for some types of reflexes, while for others a more moderate response is optimal. As such, for each item the optimal response is described, and any other response is coded as nonoptimal, ${ }^{8}$ for a maximum count of 15 nonoptimal reflexes.

The NNNS hypertonicity subscore captures increased active and passive muscle tone in the arms, legs, and trunk and summary scores of tone and movement overall. A high score indicates an infant who is consistently hypertonic in the arms, legs, trunk, neck, and shoulders. ${ }^{8}$

\section{Neuroimaging}

A pediatric neuroradiologist (B.P.S.) blinded to the patient's clinical course measured the ventricular size indices on the last HUS prior to surgical intervention, or if no surgical intervention was performed, the HUS with the worst ventriculomegaly. Measurements included left and right ventricular index (VI), anterior horn width (AHW), thalamooccipital distance (TOD), and ventricle/brain (V/B) ratio. Doppler resistive indices (RIs) were recorded in the anterior cerebral artery, both with and without gentle manual pressure on the transducer. White matter injury in the form of PVHI or cystic changes (correlating with periventricular leukomalacia [PVL] grades II-IV ${ }^{14}$ ) were described as present or absent.

The posterior fossa was assessed with MRI as clinically indicated, with MRIs obtained on 14/21 patients. The degree of fourth-ventricle dilatation was qualitatively scored $(0=$ none; $1=$ mild, ventricle compresses vermis only; 2 moderate, ventricle compresses dorsal brainstem; 3 = severe, ventricle compresses ventral brainstem against clivus; $4=$ massive, ventricle extends superiorly to supratentorial compartment), and the anteroposterior (AP) diameter of the cerebellum was measured from the fastigial point to the posterior vermis (pyramid).

\section{Early Neurodevelopmental Evaluations}

Infants who underwent neonatal NNNS and neuroimaging assessments in the neonatal period were seen in early clinical follow-up visits at 3-6 months at the Infant Neurodevelopmental Center at the Kennedy Krieger Institute, the main location for follow-up visits for high-risk NICU graduates. A visit at either 3 or 6 months was accepted, and the latter was selected if the infant had both available. We selected the comprehensive Capute Scales as the primary developmental assessment. They are a validated and standardized measure of development, both as a stand-alone measure in conditions such as antenatal ventriculomegaly ${ }^{22}$ and prematurity ${ }^{7}$ and in correlation with and prediction of future scores for the Bayley II Scales of Infant Development. ${ }^{47}$

Two separate scores are generated with the Capute Scales: an age level in months for visual and nonverbal problem-solving skills (CAT) and an age level in months for expressive and receptive language (CLAMS). Age 
TABLE 1. Clinical characteristics of 21 neonates with PHVD and developmental outcome assessment at 3-6 months from term

\begin{tabular}{lc}
\hline & Values \\
\hline Gestational age at birth & 26 wks 2 days \\
& $(25$ wks-28 wks 4 days $)$ \\
\hline Birth weight, g & $880(750-1400)$ \\
\hline 5 -min APGAR & $7(4.75-8)$ \\
\hline Treated for culture-positive/-negative sepsis & $15(71)$ \\
\hline Female sex & $9(43)$ \\
\hline Systemic steroids & $4(19)$ \\
\hline Adequate prenatal betamethasone* & $14(67)$ \\
\hline Chorioamnionitis & $1(5)$ \\
\hline Severe ROP† & $11(52)$ \\
\hline Moderate/severe BPD $\ddagger$ & $16(76)$ \\
\hline Race & $12(57)$ \\
\hline White & $7(33)$ \\
\hline African American & $1(5)$ \\
\hline Asian & $1(5)$ \\
\hline Hispanic & \\
\hline NEC stage§ & $3(14)$ \\
\hline BB & $1(5)$ \\
\hline 3B & $3(14)$ \\
\hline IVH grade & $2(10)$ \\
\hline 2 & $9(43)$ \\
\hline 3 & $7(33)$ \\
\hline (PVHI) & \\
\hline
\end{tabular}

Values are presented as median (IQR) or number (\%) of patients.

${ }^{*}$ Completed 24 hours in utero after second dose.

† Stage 3 or surgical intervention/biologic medication.

$\ddagger$ Per National Institute of Child Health and Human Development criteria ${ }^{27}$ moderate $\mathrm{BPD}$ as $\mathrm{O}_{2}$ administration for $\geq 28$ days plus treatment with $<30 \%$

$\mathrm{FiO}_{2}$ at 36 weeks PMA and severe $\mathrm{BPD}$ as $\mathrm{O}_{2}$ administration for $\geq 28$ days plus $\geq 30 \% \mathrm{FiO}_{2}$ and/or positive pressure at 36 weeks' PMA.

$\S$ Modified Bell Staging Criteria for Necrotizing Enterocolitis ${ }^{29}$; only present cases and grades displayed.

equivalents on each scale are established based on the patient's mastery of all items at the highest age level plus the addition of weighted point values for items achieved above this age level and until a ceiling is reached (i.e., failure to achieve any items in a given age level). Scores can be calculated for chronological age (the age in months from birth) or age corrected for degree of prematurity (corrected age; determined by subtracting the number of weeks born preterm before 40 weeks from the chronologic age). In this study corrected age was used to calculate the quotients below.

Developmental quotients (DQs) for the CLAMS and CAT were calculated as follows: CLAMS or CAT DQ = (age equivalent on CLAMS or CAT/age corrected for degree of prematurity) $\times 100$. Scores at the 3 - and 6-month time points are comparable to each other, as DQs are adjusted per age in this way, with 100 as a perfect score, or fully "on track" for age.
TABLE 2. Age characteristics of 21 neonates at the time of imaging and developmental assessment

\begin{tabular}{lc}
\hline \multicolumn{2}{l}{ Values } \\
\hline PMA & \\
\hline At HUS & 31 wks 6 days \\
& $(30$ wks-33 wks 6 days $)$ \\
\hline At MRI & $37.5(35.5-40.4)$ \\
\hline At NNNS & $37.4(34.4-39.6)$ \\
\hline Median age at follow-up exam (mos) & \\
\hline Chronological age & $7.0(6.0-9.0)$ \\
\hline Age corrected for prematurity & $4.0(3.0-5.5)$ \\
\hline NNNS exams performed in PMA category & $2(10)$ \\
\hline$<34$ wks & $4(19)$ \\
\hline $34.0-34.6$ wks & $1(5)$ \\
\hline $35.0-35.6$ wks & $3(14)$ \\
\hline $36.0-36.6$ wks & $11(52)$ \\
\hline $37+$ wks &
\end{tabular}

Values are presented as median (IQR) or number (\%) of patients.

MQs were calculated as follows: $\mathrm{MQ}=$ (motor age/age corrected for degree of prematurity) $\times 100$.

\section{Statistical Analyses}

Descriptive statistics, including medians, standard deviations, interquartile ranges, and percentages, were used to summarize the cohort. Logistic regressions were used to examine the relationships between imaging parameters, neurosurgical interventions, and NNNS examination subscores with MQs less than 50 and CAT and CLAMS DQs less than 85, both corrected for degree of prematurity. Measured covariates included infant demographic and clinical characteristics (Table 1) and PMA at the time of NNNS assessment and HUS and MRI examinations during the neonatal period (Table 2). Analyses were not adjusted for these potential confounders, given the small sample size. All analyses were conducted in $\mathrm{R}$ version 3.5.0 (R Foundation for Statistical Computing).

\section{Results}

\section{Patient Characteristics}

A total of 21 consented patients had NNNS and HUS examinations and follow-up Capute Scales and MQ assessments; furthermore, of these 21 patients, 14 also had MRI studies performed. The median gestational age at birth was 26.2 weeks (IQR 25.0-28.4) and median birth weight was $880 \mathrm{~g}$ (IQR 750-1400) (Table 1). The median PMAs at the times of assessment were 31.6 weeks for HUS, 37.4 weeks for MRI, and 37.4 weeks for NNNS (Table 2). The median corrected age at Capute Scales and MQ assessment was 4 months (Table 2). The 7 infants with missing follow-up metrics had no statistically significant differences from infants with complete follow-up, with the exception of maternal insurance status; mothers with private insurance had statistically significantly higher rates of infant follow-up than mothers with public insurance $(\mathrm{p}=0.029)$. 
TABLE 3. Odds ratios for 3-6-month MQs and CAT and CLAMS scores for corrected age by neurosurgical intervention, neonatal radiology, and NNNS motor and cognitive subscores

\begin{tabular}{|c|c|c|c|c|c|c|c|c|c|}
\hline \multirow[b]{2}{*}{ Predictor } & \multicolumn{3}{|c|}{ OR for $M Q<50$ (vs $\geq 50)$} & \multicolumn{3}{|c|}{ OR for CAT <85 (vs $\geq 85)$} & \multicolumn{3}{|c|}{ OR for CLAMS <85 (vs $\geq 85$ ) } \\
\hline & Estimate & $95 \% \mathrm{Cl}$ & p Value & Estimate & $95 \% \mathrm{Cl}$ & p Value & Estimate & $95 \% \mathrm{Cl}$ & $p$ Value \\
\hline Neurosurgical intervention & 16.00 & $(0.75,85.31)$ & 0.085 & 11.20 & $(1.04,120.36)$ & 0.046 & 1.07 & $(0.97,1.18)$ & 0.196 \\
\hline \multicolumn{10}{|l|}{ Neonatal radiology parameter } \\
\hline VI & 1.09 & $(0.99,1.40)$ & 0.062 & 1.12 & $(0.98,1.28)$ & 0.101 & 1.05 & $(0.93,1.18)$ & 0.433 \\
\hline AHW & 1.13 & $(1.01,1.39)$ & 0.041 & 1.12 & $(1.00,1.27)$ & 0.059 & 1.07 & $(0.97,1.18)$ & 0.196 \\
\hline TOD & 1.07 & $(0.99,1.16)$ & 0.102 & 1.05 & $(0.98,1.12)$ & 0.185 & 1.03 & $(0.97,1.10)$ & 0.345 \\
\hline V/B ratio & 2.52 & $(0.94,12.51)$ & 0.062 & $\infty$ & $(0.11, \infty)$ & 0.072 & 327.82 & $(0.21, \infty)$ & 0.122 \\
\hline $\mathrm{RI}$ (w/ pressure) & $31,564.91$ & $(0.09, \infty)$ & 0.130 & 24.19 & $(0.00, \infty)$ & 0.470 & 16.45 & $(0.00, \infty)$ & 0.522 \\
\hline RI (w/o pressure) & 6711.19 & $(0.23, \infty)$ & 0.083 & 5432.58 & $(0.07, \infty)$ & 0.135 & 31.31 & $(0.00, \infty)$ & 0.497 \\
\hline PVHI & 2.40 & $(0.28,12.46)$ & 0.515 & 9.17 & $(1.15,73.24)$ & 0.037 & 1.35 & $(0.21,8.62)$ & 0.751 \\
\hline Cerebellum AP diameter & 0.89 & $(0.74,1.09)$ & 0.261 & 0.86 & $(0.69,1.06)$ & 0.162 & 0.93 & $(0.78,1.12)$ & 0.457 \\
\hline 4th-ventricle dilatation & 3.03 & $(0.78,11.70)$ & 0.108 & 2.08 & $(0.70,6.16)$ & 0.189 & 2.19 & $(0.73,6.53)$ & 0.162 \\
\hline Cystic changes & 2.50 & $(0.18,11.72)$ & 0.718 & 12.00 & $(1.02,141.34)$ & 0.048 & 1.05 & $(0.93,1.18)$ & 0.433 \\
\hline \multicolumn{10}{|l|}{ NNNS motor exam z score } \\
\hline Excitability & 1.21 & $(0.58,2.95)$ & 0.513 & 1.47 & $(0.66,3.28)$ & 0.349 & 1.59 & $(0.71,3.60)$ & 0.263 \\
\hline Lethargy & 1.39 & $(0.63,8.64)$ & 0.204 & 1.57 & $(0.46,5.35)$ & 0.473 & 1.57 & $(0.46,5.35)$ & 0.473 \\
\hline NOR & 2.46 & $(1.15,37.57)$ & 0.034 & 3.95 & $(1.04,15.03)$ & 0.044 & 19.54 & $(1.26,302.76)$ & 0.034 \\
\hline Asymmetric reflexes & 0.89 & $(0.41,1.84)$ & 0.714 & 1.02 & $(0.51,2.04)$ & 0.959 & 1.86 & $(0.85,4.11)$ & 0.123 \\
\hline Hypertonicity & 1.68 & $(1.04,3.78)$ & 0.037 & 1.81 & $(0.97,3.37)$ & 0.060 & 1.81 & $(0.97,3.37)$ & 0.060 \\
\hline Hypotonicity & 1.66 & $(0.87,10.49)$ & 0.083 & 2.15 & $(0.71,6.53)$ & 0.176 & 2.15 & $(0.71,6.53)$ & 0.176 \\
\hline Movement & 1.59 & $(0.56,2.97)$ & 0.558 & 0.8 & $(0.37,1.73)$ & 0.576 & 1.03 & $(0.48,2.20)$ & 0.949 \\
\hline \multicolumn{10}{|l|}{ NNNS cognitive exam z score } \\
\hline Habituation & 0.77 & $(0.28,1.40)$ & 0.259 & 0.63 & $(0.28,1.40)$ & 0.259 & 0.85 & $(0.41,1.76)$ & 0.67 \\
\hline Attention & 0.67 & $(0.12,1.19)$ & 0.096 & 0.68 & $(0.27,1.71)$ & 0.407 & 0.38 & $(0.12,1.19)$ & 0.096 \\
\hline Handling & 0.89 & $(0.35,3.66)$ & 0.839 & 1.17 & $(0.37,3.67)$ & 0.792 & 1.62 & $(0.49,5.39)$ & 0.429 \\
\hline Self-regulation & 0.52 & $(0.19,1.56)$ & 0.257 & 0.49 & $(0.17,1.41)$ & 0.185 & 0.48 & $(0.17,1.39)$ & 0.177 \\
\hline Arousal & 1.69 & $(0.77,4.77)$ & 0.165 & 1.85 & $(0.76,4.49)$ & 0.176 & 1.46 & $(0.64,3.33)$ & 0.365 \\
\hline Stress/abstinence & 0.74 & $(0.37,2.57)$ & 0.952 & 2 & $(0.71,5.66)$ & 0.191 & 1.89 & $(0.68,5.27)$ & 0.222 \\
\hline
\end{tabular}

Boldface type indicates statistical significance.

\section{Early Motor Milestone Attainment Assessed by MQ}

Corrected age MQs were significantly associated with NNNS NOR and hypertonicity subscores and AHW (Table 3). Nonoptimal reflexes were observed in all 21 infants. The mean standardized $z$ score of 0.48 for the NOR subscore was calculated from the mean value of 4.5 (2.07). A 1-SD increase in the NOR subscore was related to an increase in the odds of an MQ $<50$ for corrected age (OR $2.46,95 \%$ CI 1.15-37.6, $\mathrm{p}=0.034)$. A 1-SD increase in the hypertonicity subscore was similarly related to an increase in the odds of MQ < 50 (OR 1.68, 95\% CI 1.04-3.78, p = $0.037)$. A 1-SD increase in one measure of ventricular enlargement, AHW, was also associated with increased odds of MQ < 50 (OR 1.13, 95\% CI 1.01-1.39, $\mathrm{p}=0.041)$. Need for neurosurgical intervention, and all other neuroimaging measures of ventricular size and white matter damage, were not statistically significantly associated with MQs. The negative associations between MQ for corrected age and progressively higher NOR and hypertonicity subscore $\mathrm{z}$ scores and AHW are shown in Fig. 1.

\section{Summary of Capute Scores}

A significant percentage of infants had the following abnormal follow-up measurements (all for corrected age): 1) median CAT score 93.6 (IQR 80.9-137.0), with $38 \%$ of infants having a CAT DQ $<85$; 2) median CLAMS score 97.8 (IQR 65.0-110.7), with 38\% of infants having a CLAMS DQ of $<85$.

\section{Early Foundational Visual and Problem-Solving Capute Scores}

CAT DQs $<85$ for corrected age were significantly associated with history of neurosurgical intervention, PVHI, cystic changes, and NNNS NOR subscores, and later odds of having CAT DQs $<85$ for corrected age were significantly increased by the need for neurosurgical intervention (OR 11.2, 1.04-120.36, $\mathrm{p}=0.046$ ), presence of PVHI (OR 9.17, 1.15-73.24, $\mathrm{p}=0.037)$, and cystic changes (OR $12.0,1.02-141.34, \mathrm{p}=0.048$ ) (Table 3). Those infants with any neurosurgical intervention (subgaleal or ventriculo- 

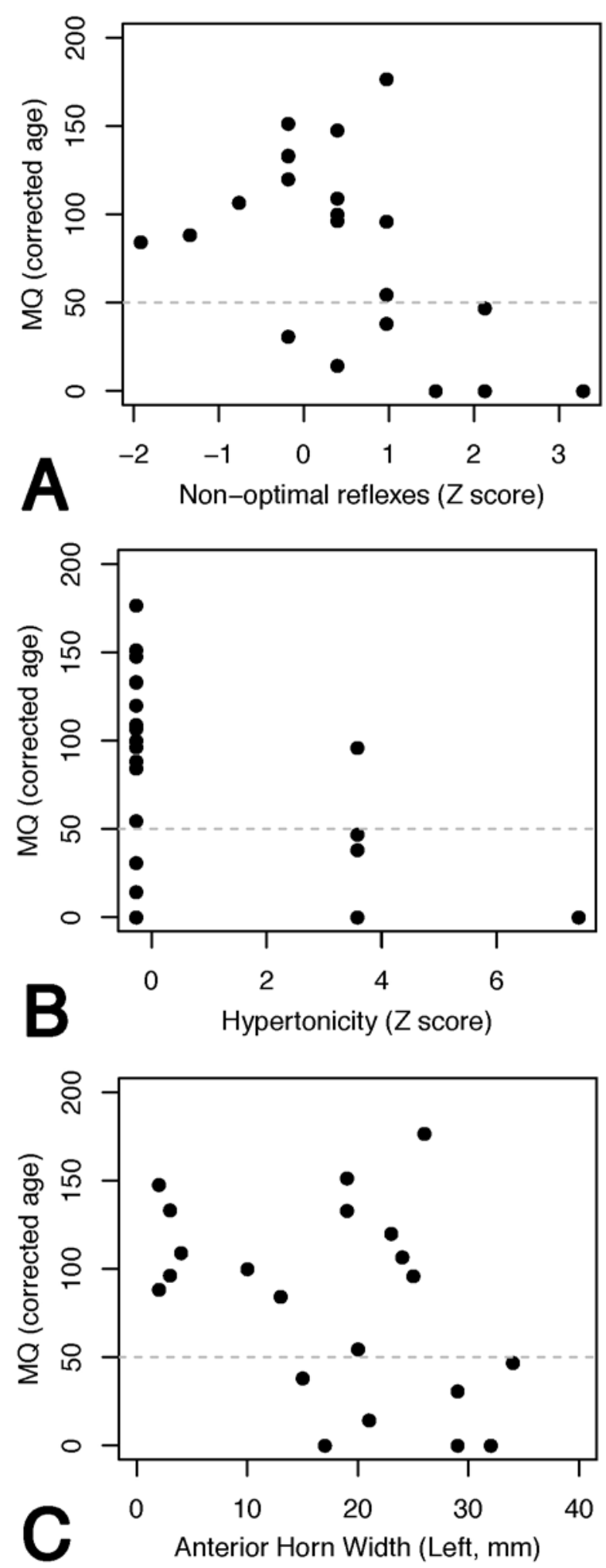

FIG. 1. Associations between continuous corrected age $M Q$ scores and NNNS NOR (A) and hypertonicity z scores (B) and AHW (C). Horizontal dotted line shows cutoff of 50 for $M Q$ scores (MQs $<50$ reflect moderate to severe delays in early motor milestone attainment). peritoneal shunts), in comparison with infants without intervention, also had significantly lower mean CAT DQs for corrected age when analyzed in a continuous fashion $(66.8$ vs $112.5, p=0.004)$. CAT DQs for corrected age were also associated with the NNNS NOR subscore, where a 1-SD increase in the NOR subscore was related to an increase in the odds of a CAT DQ $<85$ for corrected age (OR 3.95, $1.04-15.03, p=0.044)$. The negative associations between CAT DQ corrected age and progressively higher NOR subscores and presence of cystic changes, PVHI, and neurosurgical intervention is shown in Fig. 2.

\section{Early Foundational Language Capute Scores}

Corrected age CLAMS DQs $<85$ were significantly associated only with the NNNS NOR subscore. For the corrected age Capute CLAMS DQ, a 1-SD increase in the NOR subscore increased odds of having a CLAMS $\mathrm{DQ}<85$ (OR 19.5, 95\% CI 1.26-303, $\mathrm{p}=0.034$; Table 3). The negative linear association between CLAMS DQ for chronological age and progressively higher NOR z scores is shown in Fig. 3.

The odds of worse CLAMS scores at 3 or 6 months were not increased by neurosurgical intervention, measurements of ventricular size, PVHI, cystic changes, or NNNS subscores.

\section{Important Covariates}

In our previous study, we noted that specific NNNS subscores were associated with medical morbidities; namely, that infants with severe ROP had higher NNNS excitability subscores and those with severe BPD had higher NNNS NOR and lower movement subscores.$^{17}$ Here, in an analysis of a subsample of 21 participants with available developmental outcomes (i.e., MQ and Capute Scales results), BPD, NEC, and ROP were not related to corrected age MQs, CAT DQs, or CLAMS DQs. Grade of IVH using the Papile scoring system was also not related to CAT or CLAMS DQs $<85$ or MQs $<50$ for corrected age.

\section{Discussion}

Infants with IVH and those with PHVD and PHH are at risk for poor neurodevelopmental outcomes. We need to better understand the structural and functional relationships that are driving the deficits seen in follow-up. Early neurodevelopmental assessment provides evidence of functional impairment that can be interpreted alongside structural radiological markers of brain injury. Long-term follow-up continues to be important for understanding outcomes in this population. However, the initiation of focused therapeutic early interventions, as soon as or even before milestone delay is detected, has the potential to improve functional outcomes after brain injury. ${ }^{19,36}$

In our present analysis of a small group of 21 preterm patients, we found that some measures of neurological findings on neonatal neurobehavioral examinations and brain injury on neuroimaging studies correlated with early motor milestone attainment, visual and problem-solving skills, and language skills. The NNNS NOR subscore correlated with all measures-motor milestone attainment, visual and problem-solving skills, and language. The 

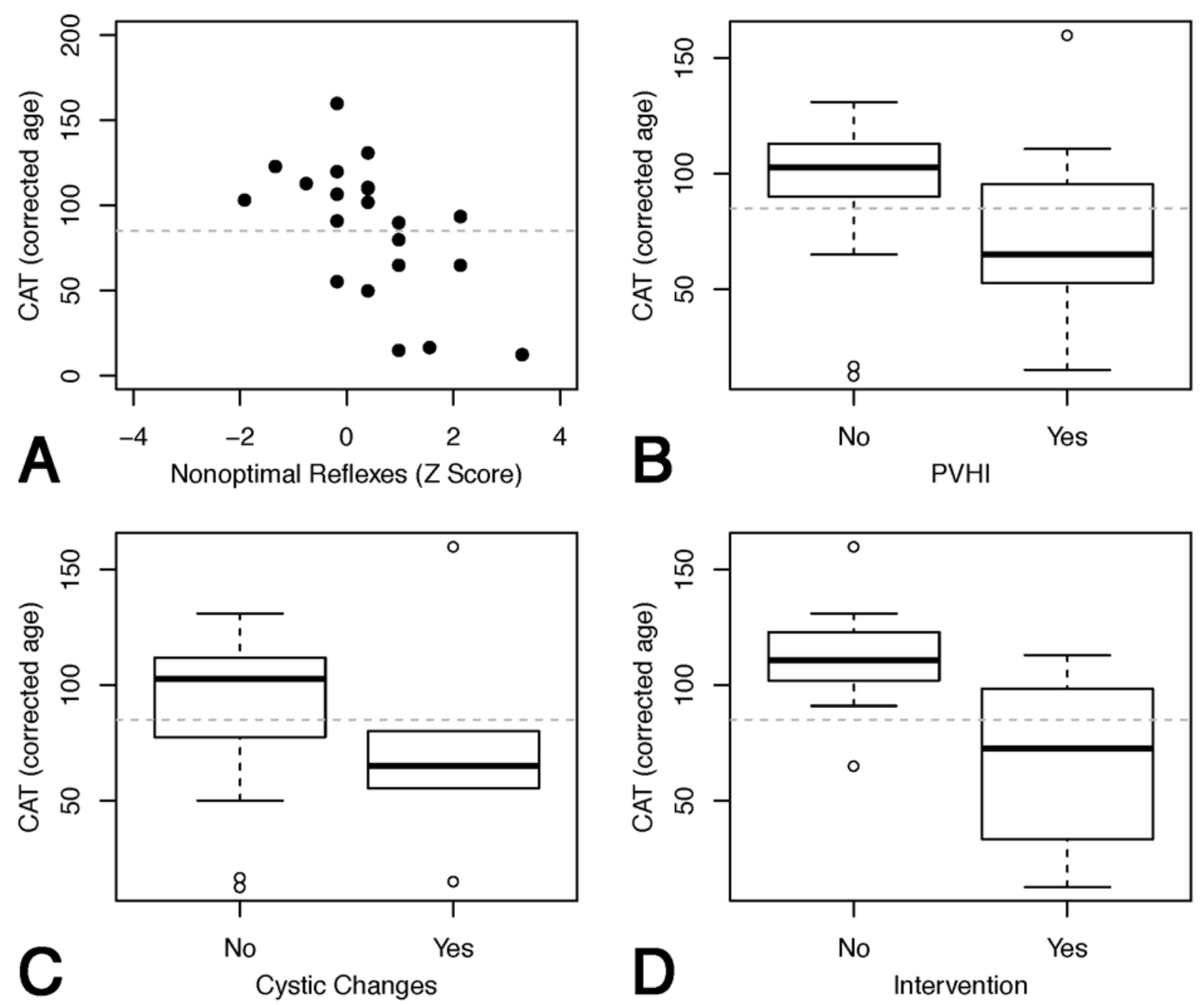

FIG. 2. Box-and-whiskers plots showing correlations between continuous corrected age Capute CAT scores and NNNS NOR z scores (A) and presence or absence of PVHI (B), cystic changes (C), and neurosurgical intervention (D). Horizontal dotted line shows the cutoff of 85 for CAT scores (CAT scores $<85$ reflect delays in early visual and problem-solving abilities).

NNNS hypertonicity subscore was associated with motor milestone attainment only. Presence of white matter injury, in the form of either cystic changes or PVHI, correlated with abnormal visual and problem-solving skills, as did need for neurosurgical intervention. Of particular note, only one ventricular size measure, AHW, was correlated with a 3-6-month developmental outcome, namely, motor milestone attainment.

\section{Neonatal Neurobehavioral Exam Correlations With 3-6-Month Outcomes}

For prediction of early developmental outcomes at 3-6 months, the neonatal NNNS NOR subscore was the only measure, among both radiological and neurological examination findings, that was correlated with all 3 outcomes: motor milestone attainment, visual and problem-solving abilities, and cognitive attainment. The NNNS hypertonicity subscore was correlated with motor milestone attainment alone.
The NNNS NOR subscore reflects neurological integrity by quantifying the number of nonoptimal reflexes. The total number of nonoptimal reflexes might be more important than any single reflex ${ }^{8}$ as the number gives a global sense of the infant's neurological status. It logically follows that the number of nonoptimal reflexes in the neonatal period would correlate with pure motor milestone attainment at 3-6 months, because the motor milestone delays persisted over time. This finding has been replicated in previous studies in which higher percentages of early delay in gross motor milestone attainment had high specificity and positive predictive values for future CP. ${ }^{4}$

The number of nonoptimal reflexes also correlated with early visual and problem-solving measures. These measures in infants at 3-6 months include supporting the body on the forearms in a prone position at 3 months, manipulating fingers and hands at midline and supporting self on wrists in a prone position at age 4 months, transferring an object between the hands at age 5 months, and lifting a cup and performing a radial rake (i.e., use of all fingers 


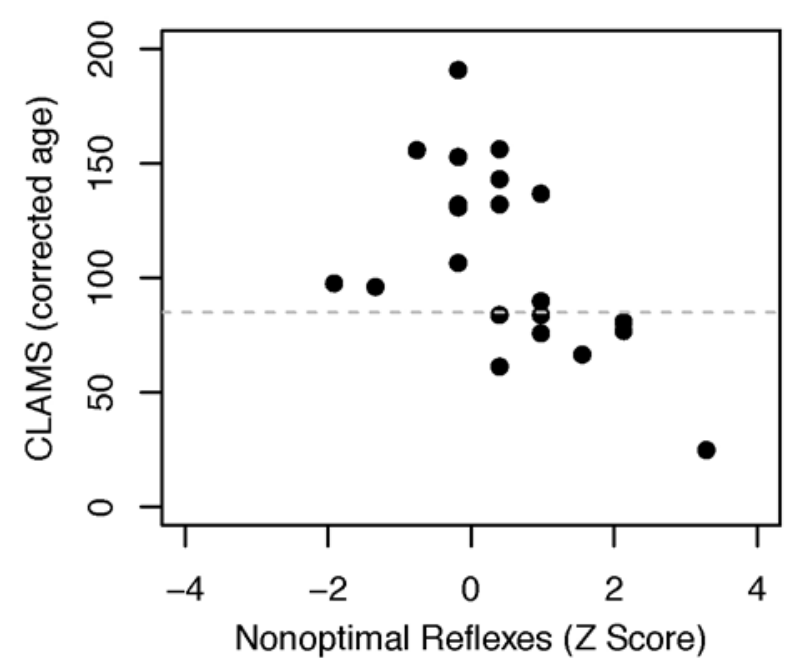

FIG. 3. Correlations between continuous corrected age Capute CLAMS scores and NNNS NOR z scores. Horizontal dotted line shows cutoff of 85 for CLAMS scores (CLAMS scores < 85 reflect delays in early language).

to sweep an object toward the palm for grasping) at age 6 months. ${ }^{1}$ These early visual and problem-solving skills are dependent on postural control, which depends on foundational motor skills. Hence poor motor skills as measured by number of nonoptimal reflexes may be a precursor for poor postural control and subsequent poor visual tracking and visual-motor coordination.

Importantly, delayed postural control might result in missed opportunities for exploration and may increase risks for not only motor but also language, social, and cognitive delays. ${ }^{6}$ Early foundational language skills assessed on the Capute CLAMS at 3 to 6 months include cooing at 3 months, orienting to voice at 4 months, orienting to a bell at 5 months, and making "ah-goo" and "razzing" sounds and babbling at 6 months. An early inability to fixate on faces would impair sound and word learning and social interaction and then further impair joint development of attention, language, social-emotional, and cognitive skills.

The NNNS NOR subscore in this cohort was a neonatal marker of poor neurological integrity that correlated with sequelae of deficits in multiple domains dependent on those motor skills, including visual attention and early language skills. By necessity, most standardized instruments of early cognitive and language development, including the Capute Scales, are greatly dependent on intact motor skills and speed of motor function. Reflex patterns require integration into later voluntary behavior to allow for development of additional skills. For example, the palmar grasp reflex is present in the newborn period but quickly disappears in the first 6 months of life to allow infants to release and then transfer objects, all required for object exploration and later problem solving. The abnormal persistence of these neonatal reflexes has long been considered a sign of CNS dysfunction. ${ }^{11}$ It is a natural extension that abnormalities in this pattern, even in the neonatal period, would correlate not just with motor, but also with cognitive and behavioral outcomes.

\section{Neonatal White Matter Injury (PVHI and Cystic Changes) Correlations With 3-6-Month Outcomes}

In the present study of infants with PHVD and PHH, PVHI was associated with 9.2 times higher odds of abnormal scores and cystic changes (PVL grades II-IV ${ }^{14}$ ) with 12 times higher odds of abnormal scores $(<85)$ for visual and problem-solving skills (Capute CAT).

In this cohort of children with PHVD, 7 infants had both PVHI and cystic changes and 2 infants had only PVHI. Worse scores on visual and problem-solving skills in children with structural damage like PVHI and PVL may result from damage to crucial brain areas in both motor function and visual processing. Visual challenges, including cortical visual impairment, are also associated with severe cystic PVL ${ }^{23,26}$; these visual challenges may also account for lower CAT scores in this group as poor visual perception skills may affect the CAT items.

These poor 3-6-month CAT scores may function as precursors for later motor and cognitive developmental delays. Previous studies have shown higher rates of both gross motor and cognitive problems in infants with both PVHI and cystic changes/PVL. One study found an $80 \%$ prevalence of $\mathrm{CP}$ in a group of preterm infants with $\mathrm{PVHI}$ with shunts, but no cases of CP in those with grade III IVH with shunts and without PVHI. ${ }^{46}$ The rate of severe CP additionally increases with grade of PVL; although $10 \%$ of children with grade I PVL develop spastic diplegia by school age, the percentage increases to almost $50 \%$ in children with cystic PVL. ${ }^{14}$ More than $50 \%$ of children with PVHI are diagnosed with intellectual disability at school age $^{24}$ and are at higher risk for clinical and subclinical behavior problems.

In our cohort we did not find any 3-6-month Capute CLAMS language correlates of antecedent PVHI or cystic changes, but this correlation may become more obvious at later time points when the demands for language comprehension and expression become more substantial.

\section{Ventricle Size and Later Neurodevelopmental Outcomes}

None of the 3 ventricle size measures (V/B ratio, AHW, VI) were correlated with early visual and problem-solving scores or language on the Capute Scales. Only AHW was associated with early motor milestone attainment on MQs.

An increase in AHW has been suggested to be a more sensitive marker for early worsening of hydrocephalus and is seen clinically as rounding of the frontal horns. Both lower $(>6 \mathrm{~mm})$ and higher $(>10 \mathrm{~mm})$ AHW size cutoffs for surgical intervention have been used,,$^{15,30}$ but increases as small as $1 \mathrm{~mm}$ can lead to progressive decreases in motor scores at 2 years. ${ }^{28}$ Of note, nonoptimal reflexes as measured by the NOR subscore were correlated with ventricular size in the neonatal period, ${ }^{17}$ but on the Capute Scales only the reflexes subscore was associated with all 3-6-month outcomes.

\section{Neurosurgical Intervention and Later Neurodevelopmental Outcomes}

Lastly, the need for neurosurgical intervention in the neonatal period was also associated with abnormal visual and problem-solving scores on the Capute Scales assess- 
ments. Infants with PHVD and PHH who require shunt placement are at greater risk for most adverse neurodevelopmental outcomes than those with only PHVD.,25 Younger age at permanent shunt insertion is associated with a higher risk of subsequent procedures with their inherent morbidity, ${ }^{40}$ yet recent evidence suggests that a higher ventricle size threshold for intervention is associated with MRI evidence of a more severe degree of brain injury. ${ }^{13}$ More evidence is needed to delineate the role of neurosurgical intervention in early neonatal outcomes in PHH.

\section{Limitations}

There are limitations to the present study, namely small sample size and early infant outcome measurement. First, our sample size of 21 infants is small, but to our knowledge it is the largest of its kind for these infants with shunts and severe brain injury. As such, inclusion of all potential covariates was not feasible and we could not evaluate the cumulative impact of measured comorbidities on early Capute Scales and MQ scores. However, we did find that BPD, NEC, and ROP were not related to MQ, CAT, or CLAMS scores for corrected age in this cohort. Second, our outcomes were measured at 3-6 months of age. Although these outcomes are early, earlier outcome assessment provides an opportunity for deficit-specific early intervention. These assessments at 3-6 months using the Capute Scales function as the initial description of an infant's developmental milestone trajectory; we will continue to follow these children longitudinally. Of note, however, early results for Capute Scales assessments have been shown to correlate with results for future Bayley II Scales of Infant Development. ${ }^{48}$ Lastly, the posterior fossa was assessed by MRI in only 14 of 21 patients. As such, the impact of cerebellar hemorrhage, hippocampal volumes, and other important radiological correlates with later developmental outcomes ${ }^{44,50}$ could not be completely assessed.

\section{Conclusions}

This small study of preterm infants with PHVD and PHH found that neonatal neurobehavioral measures were more consistently associated with 3-6-month early motor milestone attainment, visual and problem-solving skills, and language skills than traditional radiological measures like neonatal white matter injury, ventricle size, or grade of IVH. We particularly chose this set of radiological parameters that are accessible in most clinical settings. This work strongly suggests that neonatal neurodevelopmental examinations are more important for estimation of risk for future deficits in infants with PHVD and PHH than radiological assessments like ventricular size.

The combination of examinations with imaging has the promise to inform prompt intervention and counseling of families much earlier than is typical for these patients. Future multisite studies with larger sample sizes can begin to address the creation of a novel composite evaluation to better stratify risk outcomes in the clinical setting. Finally, this work is part of a longitudinal study; as this population is at very high risk for neurodevelopmental disability, we plan to report follow-up beyond infancy to include specific neurodevelopmental disability diagnoses.

\section{Acknowledgments}

This project was supported with funding by grants from the Thomas Wilson Sanitarium for Children of Baltimore City (to R.A.D. and M.C.A.) and the NIH T32 Training Grant T32HL125239-03 (to R.A.D.).

\section{References}

1. Accardo PJ, Capute AJ: The Capute Scales: Cognitive Adaptive Test/Clinical Linguistic \& Auditory Milestone Scale. Baltimore: Paul H. Brookes Publishing Co., Kennedy Fellows Association, 2005

2. Adams-Chapman I, Hansen NI, Stoll BJ, Higgins R: Neurodevelopmental outcome of extremely low birth weight infants with posthemorrhagic hydrocephalus requiring shunt insertion. Pediatrics 121:e1167-e1177, 2008

3. Alan N, Manjila S, Minich N, Bass N, Cohen AR, Walsh M, et al: Reduced ventricular shunt rate in very preterm infants with severe intraventricular hemorrhage: an institutional experience. J Neurosurg Pediatr 10:357-364, 2012

4. Allen MC, Alexander GR: Screening for cerebral palsy in preterm infants: delay criteria for motor milestone attainment. J Perinatol 14:190-193, 1994

5. Allen MC, Cristofalo EA, Kim C: Outcomes of preterm infants: morbidity replaces mortality. Clin Perinatol 38:441454, 2011

6. Babik I, Galloway JC, Lobo MA: Infants born preterm demonstrate impaired exploration of their bodies and surfaces throughout the first 2 years of life. Phys Ther 97:915-925, 2017

7. Belcher HM, Gittlesohn A, Capute AJ, Allen MC: Using the clinical linguistic and auditory milestone scale for developmental screening in high-risk preterm infants. Clin Pediatr (Phila) 36:635-642, 1997

8. Boukydis CF, Bigsby R, Lester BM: Clinical use of the Neonatal Intensive Care Unit Network Neurobehavioral Scale. Pediatrics 113:679-689, 2004

9. Brouwer A, Groenendaal F, van Haastert IL, Rademaker K, Hanlo P, de Vries L: Neurodevelopmental outcome of preterm infants with severe intraventricular hemorrhage and therapy for post-hemorrhagic ventricular dilatation. J Pediatr 152:648-654, 2008

10. Capute AJ: The Capute Scales: CAT/CLAMS: Instruction Manual. Baltimore: Kennedy Fellows Association, 1996

11. Capute AJ, Accardo PJ, Vining EPG, Rubenstein JE, Harryman S: Primitive Reflex Profile: Monographs in Developmental Pediatrics. Baltimore: University Park Press, 1978, Vol 1

12. Capute AJ, Shapiro BK: The motor quotient. A method for the early detection of motor delay. Am J Dis Child 139:940942,1985

13. Cizmeci MN, Khalili N, Claessens NHP, Groenendaal F, Liem KD, Heep A, et al: Assessment of brain injury and brain volumes after posthemorrhagic ventricular dilatation: a nested substudy of the randomized controlled ELVIS trial. J Pediatr 208:191-197.e2, 2019

14. de Vries LS, Eken P, Dubowitz LM: The spectrum of leukomalacia using cranial ultrasound. Behav Brain Res 49:1-6, 1992

15. de Vries LS, Groenendaal F, Liem KD, Heep A, Brouwer AJ, van't Verlaat E, et al: Treatment thresholds for intervention in posthaemorrhagic ventricular dilation: a randomised controlled trial. Arch Dis Child Fetal Neonatal Ed 104:F70F75, 2019

16. Dorner RA, Burton VJ, Allen MC, Robinson S, Soares BP: 
Preterm neuroimaging and neurodevelopmental outcome: a focus on intraventricular hemorrhage, post-hemorrhagic hydrocephalus, and associated brain injury. J Perinatol 38:1431-1443, 2018

17. Dorner RA, Soares BP, Robinson S, Allen MC, Perin J, Burton VJ: The relationship between clinical imaging and neurobehavioral assessment in posthemorrhagic ventricular dilation of prematurity. Front Physiol 10:64, 2019

18. Dyet LE, Kennea N, Counsell SJ, Maalouf EF, Ajayi-Obe M, Duggan PJ, et al: Natural history of brain lesions in extremely preterm infants studied with serial magnetic resonance imaging from birth and neurodevelopmental assessment. Pediatrics 118:536-548, 2006

19. Eliasson AC, Holmefur M: The influence of early modified constraint-induced movement therapy training on the longitudinal development of hand function in children with unilateral cerebral palsy. Dev Med Child Neurol 57:89-94, 2015

20. Fox LM, Choo P, Rogerson SR, Spittle AJ, Anderson PJ, Doyle L, et al: The relationship between ventricular size at 1 month and outcome at 2 years in infants less than 30 weeks' gestation. Arch Dis Child Fetal Neonatal Ed 99:F209_ F214, 2014

21. Goldstein RF, Cotten CM, Shankaran S, Gantz MG, Poole WK: Influence of gestational age on death and neurodevelopmental outcome in premature infants with severe intracranial hemorrhage. J Perinatol 33:25-32, 2013

22. Graham E, Duhl A, Ural S, Allen M, Blakemore K, Witter F: The degree of antenatal ventriculomegaly is related to pediatric neurological morbidity. J Matern Fetal Med 10:258-263, 2001

23. Guzzetta A, Fiori S, Scelfo D, Conti E, Bancale A: Reorganization of visual fields after periventricular haemorrhagic infarction: potentials and limitations. Dev Med Child Neurol 55 (Suppl 4):23-26, 2013

24. Guzzetta F, Shackelford GD, Volpe S, Perlman JM, Volpe JJ: Periventricular intraparenchymal echodensities in the premature newborn: critical determinant of neurologic outcome. Pediatrics 78:995-1006, 1986

25. Holwerda JC, Van Braeckel KNJA, Roze E, Hoving EW, Maathuis CGB, Brouwer OF, et al: Functional outcome at school age of neonatal post-hemorrhagic ventricular dilatation. Early Hum Dev 96:15-20, 2016

26. Jacobson L, Ek U, Fernell E, Flodmark O, Broberger U: Visual impairment in preterm children with periventricular leukomalacia-visual, cognitive and neuropaediatric characteristics related to cerebral imaging. Dev Med Child Neurol 38:724-735, 1996

27. Jobe AH, Bancalari E: Bronchopulmonary dysplasia. Am J Respir Crit Care Med 163:1723-1729, 2001

28. Kahle KT, Kulkarni AV, Limbrick DD Jr, Warf BC: Hydrocephalus in children. Lancet 387:788-799, 2016

29. Lee JS, Polin RA. Treatment and prevention of necrotizing enterocolitis. Semin Neonatol 8:449-459, 2003.

30. Leijser LM, Miller SP, van Wezel-Meijler G, Brouwer AJ, Traubici J, van Haastert IC, et al: Posthemorrhagic ventricular dilatation in preterm infants: when best to intervene? Neurology 90:e698-e706, 2018

31. Lester BM, Tronick EZ, Brazelton TB: The Neonatal Intensive Care Unit Network Neurobehavioral Scale procedures. Pediatrics 113:641-667, 2004

32. Luu TM, Ment LR, Schneider KC, Katz KH, Allan WC, Vohr BR: Lasting effects of preterm birth and neonatal brain hemorrhage at 12 years of age. Pediatrics 123:1037-1044, 2009

33. McCurdy M, Bellows A, Deng D, Leppert M, Mahone E, Pritchard A: Test-retest reliability of the Capute Scales for neurodevelopmental screening of a high risk sample: impact of test-retest interval and degree of neonatal risk. J Neonatal Perinatal Med 8:233-241, 2015

34. McGowan EC, Vohr BR: Neurodevelopmental follow-up of preterm infants: what is new? Pediatr Clin North Am 66:509-523, 2019

35. Ment LR, Vohr B, Allan W, Westerveld M, Katz KH, Schneider KC, et al: The etiology and outcome of cerebral ventriculomegaly at term in very low birth weight preterm infants. Pediatrics 104:243-248, 1999

36. Morgan C, Novak I, Dale RC, Guzzetta A, Badawi N: Single blind randomised controlled trial of GAME (Goals - Activity - Motor Enrichment) in infants at high risk of cerebral palsy. Res Dev Disabil 55:256-267, 2016

37. Noble Y, Boyd R: Neonatal assessments for the preterm infant up to 4 months corrected age: a systematic review. Dev Med Child Neurol 54:129-139, 2012

38. O'Shea TM, Kuban KC, Allred EN, Paneth N, Pagano M, Dammann O, et al: Neonatal cranial ultrasound lesions and developmental delays at 2 years of age among extremely low gestational age children. Pediatrics 122:e662-e669, 2008

39. Papile LA, Burstein J, Burstein R, Koffler H: Incidence and evolution of subependymal and intraventricular hemorrhage: a study of infants with birth weights less than 1,500 gm. J Pediatr 92:529-534, 1978

40. Riva-Cambrin J, Kestle JR, Holubkov R, Butler J, Kulkarni $\mathrm{AV}$, Drake J, et al: Risk factors for shunt malfunction in pediatric hydrocephalus: a multicenter prospective cohort study. J Neurosurg Pediatr 17:382-390, 2016

41. Robinson S: Neonatal posthemorrhagic hydrocephalus from prematurity: pathophysiology and current treatment concepts. J Neurosurg Pediatr 9:242-258, 2012

42. Roze E, Van Braeckel KN, van der Veere CN, Maathuis CG, Martijn A, Bos AF: Functional outcome at school age of preterm infants with periventricular hemorrhagic infarction. Pediatrics 123:1493-1500, 2009

43. Spittle AJ, Orton J: Cerebral palsy and developmental coordination disorder in children born preterm. Semin Fetal Neonatal Med 19:84-89, 2014

44. Strahle JM, Triplett RL, Alexopoulos D, Smyser TA, Rogers CE, Limbrick DD Jr, et al: Impaired hippocampal development and outcomes in very preterm infants with perinatal brain injury. Neuroimage Clin 22:101787, 2019

45. Tronick EZ, Olson K, Rosenberg R, Bohne L, Lu J, Lester BM: Normative neurobehavioral performance of healthy infants on the Neonatal Intensive Care Unit Network Neurobehavioral Scale. Pediatrics 113:676-678, 2004

46. Tsai AJ, Lasky RE, John SD, Evans PW, Kennedy KA: Predictors of neurodevelopmental outcomes in preterm infants with intraparenchymal hemorrhage. J Perinatol 34:399-404, 2014

47. Visintainer PF, Leppert M, Bennett A, Accardo PJ: Standardization of the Capute Scales: methods and results. J Child Neurol 19:967-972, 2004

48. Voigt RG, Brown FR III, Fraley JK, Llorente AM, Rozelle J, Turcich M, et al: Concurrent and predictive validity of the Cognitive Adaptive Test/Clinical Linguistic and Auditory Milestone Scale (CAT/CLAMS) and the Mental Developmental Index of the Bayley Scales of Infant Development. Clin Pediatr (Phila) 42:427-432, 2003

49. Wellons JC III, Shannon CN, Holubkov R, Riva-Cambrin J, Kulkarni AV, Limbrick DD Jr, et al: Shunting outcomes in posthemorrhagic hydrocephalus: results of a Hydrocephalus Clinical Research Network prospective cohort study. J Neurosurg Pediatr 20:19-29, 2017

50. Williamson WD, Percy AK, Fishman MA, Cheek WR, Desmond MM, LaFevers N, et al: Cerebellar hemorrhage in the term neonate: developmental and neurologic outcome. Pediatr Neurol 1:356-360, 1985

\section{Disclosures}

The authors report no conflict of interest concerning the materi- 
als or methods used in this study or the findings specified in this paper.

\section{Author Contributions}

Conception and design: Dorner, Soares, Burton. Acquisition of data: Dorner, Soares, Ramos, Burton. Analysis and interpretation of data: Dorner, Allen, Robinson, Soares, Perin, Gerner, Burton. Drafting the article: Dorner, Ramos. Critically revising the article: Dorner, Allen, Robinson, Perin, Gerner, Burton. Reviewed submitted version of manuscript: all authors. Approved the final version of the manuscript on behalf of all authors: Dorner. Statistical analysis: Perin.

\section{Supplemental Information}

Previous Presentations

Portions of this work were presented in poster form at the Pediatric Academic Societies Annual Meeting, Baltimore, Maryland, April 27, 2019.

\section{Correspondence}

Rebecca A. Dorner: Bloomberg Children's Center, Baltimore, MD.rdorner1@jhmi.edu. 